\title{
SUPPLEMENTARY MOTOR AREA OF THE MONKEY'S CEREBRAL CORTEX: SHORT- AND LONG-TERM DEFICITS AFTER UNILATERAL ABLATION AND THE EFFECTS OF SUBSEQUENT CALLOSAL SECTION ${ }^{1}$
}

\author{
COBIE BRINKMAN \\ Experimental Neurology Unit, The John Curtin School of Medical Research, Australian National University, \\ Canberra, Australia 2601
}

Received March 7, 1983; Revised November 11, 1983; Accepted November 15, 1983

\begin{abstract}
The short-term and long-term behavioral effects of unilateral lesions of the supplementary motor area (SMA) were studied in five monkeys (Macaca fascicularis ssp). A monkey with a unilateral lesion of the premotor area (PM) served as a control. In all animals, general behavior was unaffected by the lesions. For a few weeks postoperatively, all monkeys showed a clumsiness of forelimb movements, bilaterally, which involved both the distal and proximal muscles. Two SMA-lesioned monkeys (but not the PM-lesioned one), studied for up to 1 year postoperatively, showed a characteristic deficit of bimanual coordination where the two hands tended to behave in a similar manner instead of sharing the task between them. This deficit was more pronounced after a lesion contralateral to the nonpreferred hand. The deficit was interpreted as indicating that the intact SMA now influenced the motor outflow of both the ipsilateral hemisphere and the contralateral one through the corpus callosum. Callosal section immediately abolished the bimanual deficit, although the clumsiness returned transiently. The results imply that SMA may give rise normally to discharges informing the contralateral hemisphere of intended and/or ongoing movements via the corpus callosum. This may prevent duplication of responses during unimanual tasks and help to coordinate the behavior of both hands during a bimanual task.
\end{abstract}

The supplementary motor area (SMA) is located in the superior frontal gyrus, on the medial side of the cerebral hemisphere, and largely coincides with Brodmann's cytoarchitectonic area 6 . In recent years, SMA has been a focus of interest in studies of the cerebral control of movement. Anatomically, it lies "upstream" of the primary motor area of the precentral gyrus, area 4 (Matsamura and Kubota, 1979; Muakassa and Strick, 1979), which seems to be one of the most important targets of SM A's efferent projections (DeVito and Smith, 1959). Recordings of the activity of SMA neurons in conscious, behaving monkeys have demonstrated that many such cells are active during movement yerformance and differ in their properties from primary motor area neurons studied under the same conditions (Brinkman and Porter, 1979; Wise and Tanji, 1981; Tanji and Kurata, 1982). Studies of changes in regional cerebral blood flow $(\mathrm{rCBF})$ in man indicate that SMA is involved in the execution of complex movement sequences (Orgogozo

\footnotetext{
This work was supported by the National Health and Medical Research Council of Australia.
}

and Larsen, 1979; Roland et al., 1980) and indeed in the planning of such sequences, since only SMA shows a relative increase in $\mathrm{rCBF}$ when subjects are asked to think about the movements but not to execute them (Roland et al., 1980). The above studies suggest that SMA may be of great importance in the programming of movements, especially through instructions relayed to the primary motor area.

However, few studies have been concerned with the effects of selective ablation of SMA, and the available evidence in monkeys does not seem to bear out this importance of SMA. A transient grasp reflex has been found (Penfield and Welch, 1951; Travis, 1955; Smith et al., 1981), and muscle tone may be affected (Travis, 1955), but extensive lesions may also fail to produce any deficits (DeVito and Smith, 1959; Coxe and Landau, 1965). In man, disease processes involving SMA may produce aphasia and disturbances of movement (Masdeu et al., 1978; Goldberg et al., 1981). However, when SMA is acutely removed, as in excision of an epileptic focus, an akinesia results which is global, i.e., bilateral, although the contralateral body is more affected; and regardless 
of the side of the excision, mutism is found (Laplane et al., 1977; Damasio and Van Hoesen, 1980). This dramatic picture disappears within a few weeks; after a few months, such patients have little movement disturbance. Nevertheless, they continue to show a deficit when simultaneous but different movements of the two hands are required (Laplane et al., 1977). A bimanual coordination deficit has also been found in monkeys with unilateral SMA ablations (Brinkman, 1981).

The present paper provides a description of the shortterm and long-term deficits after SMA lesions in the monkey and of the effects of subsequent callosal section. Short communications of some of the findings have been published elsewhere (Brinkman, 1981, 1982).

\section{Materials and Methods}

Long-term behavioral data were collected over many months from three juvenile, male crab-eating macaques (Java monkey, Macaca fascicularis ssp) born and bred in the laboratory. Two (SMX-1 and -2) received unilateral ablation of the medial part of Brodmann's area 6 , the SMA, and the third (PMX-1) received unilateral ablation of the lateral part of area 6 , the premotor area, on the convexity of the hemisphere. In SMX-1 and PMX-1 the lesions were made in the hemisphere contralateral to the nonpreferred hand, whereas in SMX-2 the lesion was contralateral to the preferred hand (for the definitions of preferred and nonpreferred, see below, under "Behavioral testing"). Short-term data over 7 days postoperatively were obtained from three more monkeys (M-6, -7 , and -9) with unilateral SMA lesions.

Surgery. The lesions were made under ketamine/xylazine anesthesia (Banknieder et al., 1978) using subpial suction with full aseptic ritual. They are shown in Figure 1. Because M-9 of the short-term animals showed the more pronounced movement deficits, and because there is disagreement over whether SMA has a clear somatotopic organization (Brinkman and Porter, 1979; Macpherson et al., 1982; Tanji and Kurata, 1982) in the longterm animals SMX-1 and -2 , the lesions were made to include SMA in its entire extent as defined in recent experiments. Rostrally, the rostral tip of the superior limb of the arcuate sulcus was used as a landmark and caudally, the posterior tip of the superior precentral sulcus. All cortex of the superior frontal gyrus on the medial surface of the hemisphere between these points was removed, as well as the upper bank of the cingulate sulcus. Laterally, the lesion included approximately 3 $\mathrm{mm}$ of cortex on the crown of the superior frontal gyrus (Fig. 1). The premotor area lesion in PMX-1 involved the entire posterior bank of the arcuate sulcus at its concavity. It extended caudally to include 3 to $4 \mathrm{~mm}$ of the cortex on the surface of the hemisphere to the approximate border of areas 6 and 4, and medially to a level just below the superior precentral sulcus (Fig. 1). The removal of cortex buried in the sulci was done by gently exposing the pial surface covering the opposite banks. Callosal section was performed in SMX-1 and -2 and in PMX-1 using fine glass pipettes and special retractors and with the aid of an automatic stereo microscope. The callosum was approached from the side op-

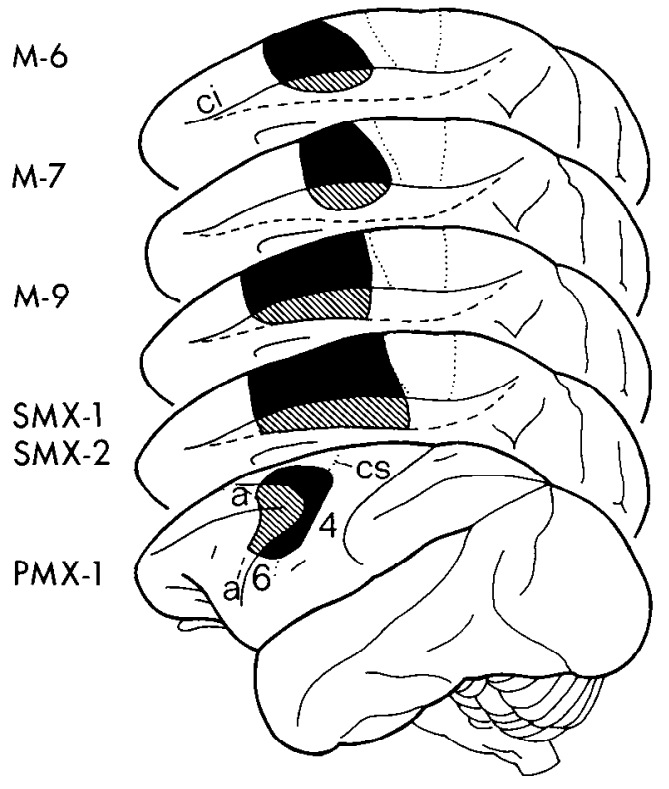

Figure 1. Lesions (black and shaded) of SMA (M-6, -7, and -9 , and SMX-1 and -2) and of PM (PMX-1). For convenience, all lesions of SMA are shown in the same side of the brain. The black areas indicate the cortical area removed on the surface of the hemisphere, and the shaded areas show cortex removed which is normally buried in the sulci: the upper bank of the cingulate sulcus $(c i)$ in SMA lesions, and the posterior bank of the arcuate sulcus $(a)$ in the PM lesions. The depth of these sulci is indicated by dashed lines. $c s$, central sulcus. The numbers 6 and 4 denote the cytoarchitectonic areas according to Brodmann, and their approximate borders are shown by the dotted lines. The lesions in M-6 and -9 have been verified histologically, and those in SMX-1 and -2 and PMX-1 are based on drawings and notes made during surgery.

posite the lesioned hemisphere and transected completely in all animals. The anterior commissure was left intact. To reduce postoperative edema, dexamethasone $(0.05 \mathrm{mg} / \mathrm{kg}$, i.m., twice daily) was administered for 1 week and then was gradually withdrawn during the second week. Antibiotics were given routinely to all animals with each surgical procedure.

Histology. Figure 1 shows the histologically verified lesions of M-6, -7 , and -9 and the intended lesions based on data and drawings made during surgery in SMX-1 and -2 and in PMX-1, which animals are still alive and kept for further study.

Completeness of the callosal section could be confirmed during surgery through exposure of the roof of the third ventricle and visualization of the pretectal commissure posteriorly and of the anterior commissure anteriorly.

The cortex buried in the sulci in these animals was removed by exposing the pial surface covering the opposite banks, with the aid of a microscope. The transient weakness of the contralateral leg seen postoperatively in SMX-1 and -2, and presumably due to local edema affecting the leg representation of area 4 , was taken to indicate that the lesion had, posteriorly, included most, if not all, of the medial part of area 6 and included the posterior margin of the SMA forelimb representation, 
while the anterior boundary of the lesion extended to a level rostral to where neurons related to forelimb movements or retrogradely labeled corticospinal neurons can be found (Brinkman and Porter, 1979; Macpherson et al., 1982, Tanji and Kurata, 1982). Thus, the lesions were in accordance with recent data which suggest that SMA extends more rostrally than is indicated in Woolsey et al.'s (1952) original map.

\section{Behavioral testing}

Behavioral testing was carried out pre- and postoperatively and included observation of general behavior, the use of an individual hand during a test requiring relatively independent finger movements, and a bimanual coordination test. Placing reactions were not tested.

General behavior. This was studied both with the animals housed individually (all monkeys) and in a gang cage with unoperated peers (SMX-1 and -2 and PMX1). It included locomotion, climbing, resting posture, grooming, social interaction, and vocalization. No formal neurological testing of reflexes or muscle tone was carried out.

Relatively independent finger movements. The monkeys were presented with a test board adapted from the test of Brinkman and Kuypers (1973) and with part of the cage wall covered with a perspex barrier which allowed the use of only one hand at a time but which left the animals full vision during the movement task. The board $(10 \times 20 \mathrm{~cm})$ contained slots, $15 \mathrm{~mm}$ long, $6 \mathrm{~mm}$ deep, and 4 to $6 \mathrm{~mm}$ wide so as to allow access to only a single digit, and oriented at $45^{\circ}, 90^{\circ}, 135^{\circ}$, and $180^{\circ}$. Slots with different angles were distributed at random over the board's surface (see Fig. 5). A small morsel of food placed at the center of a slot and not protruding above the board's surface could be retrieved only by placing the index on one side of the food and then opposing the thumb on the other side, keeping the other fingers flexed and out of the way, in a precision grip fashion (Napier, 1956). The board was presented with its shorter side facing the animal, and full extension of the limb was necessary to reach the most distant slots. All animals, like more than 40 other monkeys tested (C. Brinkman, unpublished observations; cf. Engele et al., 1982) when given choice of hands, showed a clear preference for one particular hand on this test. Movements of this hand were faster, as evidenced by the number of slots emptied during a given period; opposition movements were more coordinated; and associated movements of the middle, ring, and little fingers were absent or fewer as compared to the other hand. The former hand was called the "preferred" hand and the latter the "nonpreferred" hand, and lesions were made accordingly.

Bimanual coordination. Only SMX-1 and -2 and PMX-1 received this test. The test was adapted from that of Mark and Sperry (1968) and consisted of a raised perspex plate, $8 \mathrm{~mm}$ thick, with holes of $6 \mathrm{~mm}$ diameter drilled in a random distribution over the surface. A sticky food (currants) lodged in a hole could be retrieved only by pushing with the index of one hand while the other hand was cupped underneath to catch the falling bait. Alternatively, the bait could be pushed up from below with one hand and retrieved by the other hand as it emerged above the surface, and PMX-1 nearly always used this approach. Virtually no training was necessary for this task, and monkeys would perform in a consistent manner after only a few trials. Performance on this test and on the board with the slots was recorded pre- and postoperatively on videotapes and movie films, some of the latter at high speed ( 48 frames/sec).

\section{Results}

The consequences of unilateral SMA ablations can be divided into two periods, with short-term behavioral deficits observed mainly in the first few postoperative weeks and with long-term deficits which were present up to 1 year after surgery. Deficits in the control of relatively independent finger movements belong to the first category, bimanual coordination deficits to the second. They will be described below. A third section will deal with the effects of a lesion in the lateral part of area 6, the premotor area (PM). Last, the effects of subsequent callosal section upon these lesions will be described.

\section{Short-term effects of unilateral SMA ablation}

General behavior. No gross changes in general behavior were seen after unilateral SMA lesions. Some weakness of the contralateral leg occurred in the first postoperative week in monkeys SMX-1 and -2 which had the largest lesions, presumably because of edema affecting the leg representation area of the primary motor cortex. Apart from this brief episode, locomotion and climbing were swift and well coordinated. Resting posture was normal and symmetric except in SMX-2, with a lesion opposite the preferred hand. The affected extremity was sometimes left in an abnormal position, e.g., hanging at the animal's side, whereas the other arm rested, flexed, on the knees. A similar neglect was also seen in this animal while feeding where it used preferentially the normal hand. Other animals would often use the affected hand to take food. Release of the food into the mouth was not impaired in any animal when using the affected hand. Grooming was normal and done with either hand, and both spontaneous and elicited vocalization were present.

Unimanual food retrieval using relatively independent finger movements. Only SMX-2, with the lesion opposite the preferred hand, refused to use this hand to retrieve food from the slot board immediately postoperatively, and regular use of this hand was not seen until the second week. All other animals used either hand as required by the test situation and, upon casual inspection, seemed to show little difficulty in executing this task, except that the movements appeared to be somewhat slower and more associated movements were seen.

However, frame-by-frame analysis of videotapes and movie films, in particular the high speed ones, revealed a characteristic deficit which was present bilaterally. Preoperatively, a monkey would retrieve a bait from a slot with a swift, well coordinated opposition movement of index and thumb. During the reach for the slot, often the hand showed an anticipatory posture with the index and thumb extended and the other fingers flexed. The index as a rule was inserted into the slot first, followed by opposition of the thumb, and the food was lifted out with a combined supination and dorsiflexion movement 

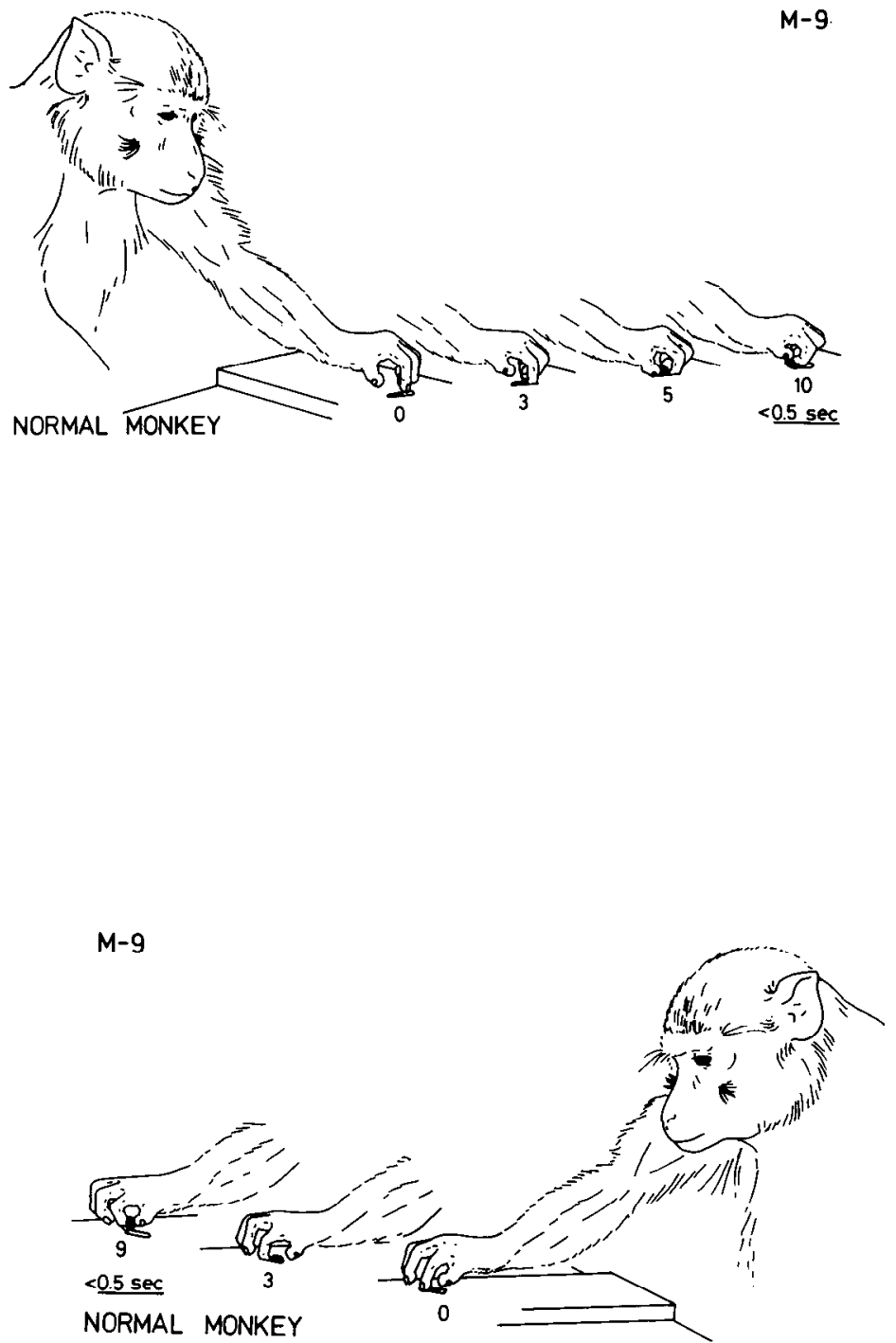

Figure 2. Drawings taken from single frames of a movie tìlm showing unimanual retrieval of a small piece of food from a narrow slot by a normal monkey. A perspex barrier prevented use of the other hand. The numbers under the figurines in this figure and in Figures 3, 4, and 6 refer to the numbers of the frames after that from which the first figurine was taken. Film speed for this figure was 24 frames/sec. The left hand of this animal showed better coordination than the right hand in the opposition of thumb and index, while the other fingers were kept flexed and out of the way. In the right hand, associated movements of these fingers occurred. When given the choice, the animal would also prefer the left hand; therefore, it was regarded as a left-handed monkey.

of the wrist (Fig. 2). Such retrieval rarely took more than $1 / 2$ sec. Postoperatively, this movement sequence appeared to be broken up. The "shaping" of the hand while reaching out, prior to retrieval, was absent. Instead, a number of small movements might occur, such as flexion and extension with abduction of the fingers; the thumb was often tucked into the palm of the hand (Fig. 4, top, 1 to 6 ). Index or thumb might be placed into a slot, but opposition of the other digit would not always follow. Rather, the digit inserted would attempt to pull or push the bait out (Fig. 3, top, $O$ to 9 ). The middle, ring, and little fingers, which in the normal monkey are kept flexed and out of the way and which do not interfere with the movements of index and thumb, would in the SMAlesioned animals assume awkward postures, often giving the hand a claw-like appearance. Figures 3 and 4 show the movements of the contralateral and ipsilateral hands of two monkeys-M-9, with a lesion opposite the preferred hand, 1 week after the lesion, and SMX-1, with a lesion opposite the nonpreferred hand, 2 weeks postoperatively. In both animals, the lesion was on the right side. Eventually, the monkeys did dislodge the food from the slots, but they typically took 1 sec or more to do so. Within this period, many different movements, such as those described above, could be seen, at times with changes occurring from one movie frame to the next, i.e., within approximately $40 \mathrm{msec}$ (Fig. 4, top, 1 to 6 ).

Thus, it seemed that, although individual components of the normal movement sequence of reaching and "precision grip" retrieval, such as thumb flexion and extension, were present, these components were not well coordinated into the total movement performance.

In all animals, this deficit was present bilaterally and affected both the contra- and ipsilateral extremity to the same extent, regardless of whether the lesion was opposite the preferred hand or not (cf. Figs. 3 and 4). In SMX-1 and -2, which were studied over periods longer than 1 week, the deficit disappeared after 2 weeks. After 4 weeks it was rarely seen, and then usually in the hand contralateral to the lesion. One year postoperatively, only an occasional "slip of the fingers" remained and was usually seen in SMX-2.

Although the monkeys were not formally tested for reaching accuracy, some suggestion that the deficit may not have been confined to the distal extremity was gained by plotting, from the movie records, the sequence of the slots emptied before an animal rested. This was done for three of the five SMA-lesioned animals. Preoperatively, the monkeys would remove the baits from the slots in a systematic manner, proceeding from one slot to the next or to one quite close to it. Postoperatively, the trajectories thus mapped showed a much more erratic course (Fig. 5). Indeed, in a frame-by-frame analysis, a monkey could be seen to steer its hand apparently to one slot but then to veer off to another (Fig. 4, top, 1 to 6 ). Not infrequently, the hand hit the edge of the perspex barrier or the $(5 \times 5 \mathrm{~cm})$ cage mesh when the animal reached out.

Thus, it seems that after a unilateral lesion of SMA a transient movement deficit results which involves both the distal and proximal extremity and which is bilateral, regardless of whether the lesion was placed contralateral to the preferred or to the nonpreferred hand.

\section{Long-term effects of unilateral SMA ablation}

These were studied for up to 1 year postoperatively in two monkeys, SMX-1 and -2. In SMX-1, the lesion was made contralateral to the nonpreferred hand and in SMX-2, contralateral to the preferred hand. In their general behavior, no changes lasted after the immediate postoperative period, but SMX-2 would sometimes leave the contralateral arm in an awkward position, especially when startled. One year after surgery, little if any evi- 


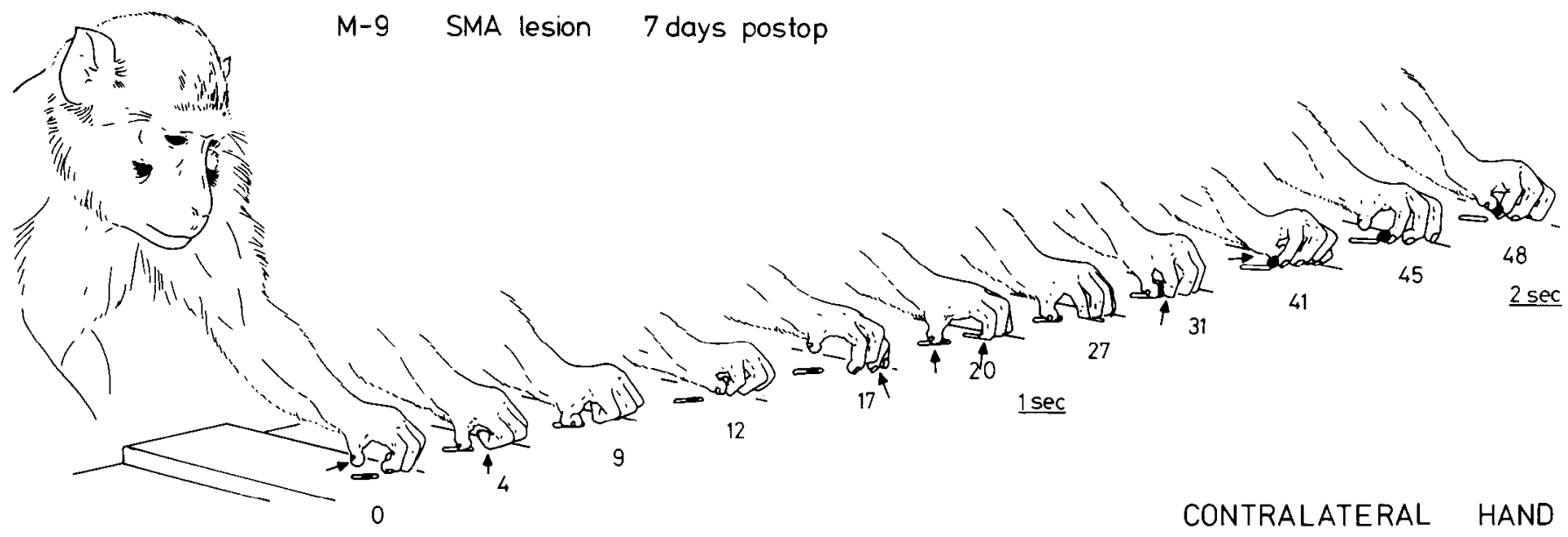

IPSILATERAL HAND

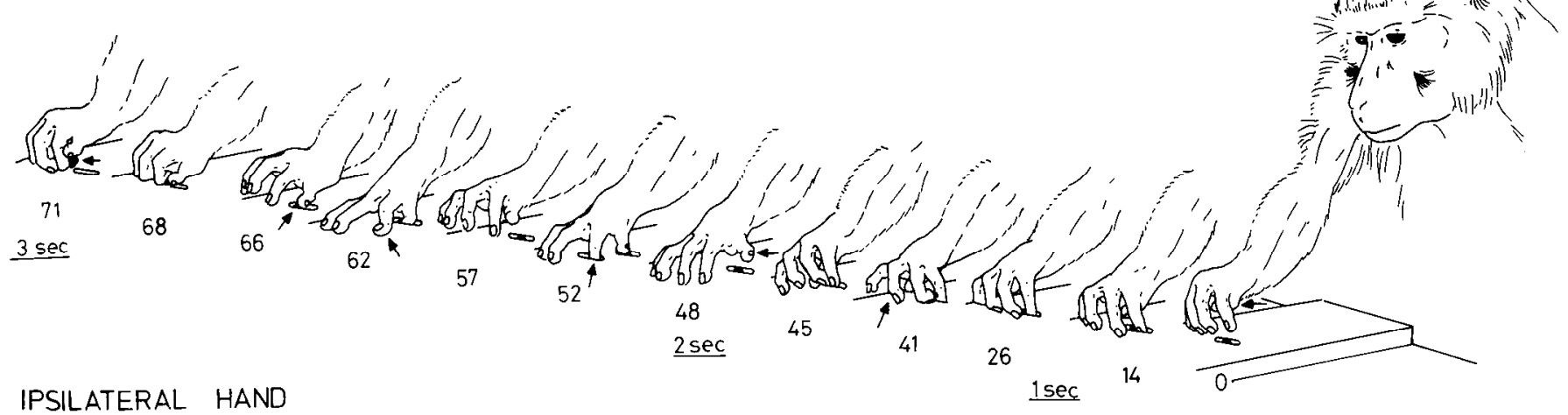

Figure 3. Performance of the same monkey as in Figure 2, 1 week after a right SMA ablation (see Fig. 1) contralateral to the preferred hand. Film speed was 24 frames/sec. The normally swift and well coordinated movements are broken up, and abnormal movements (arrows) occurred, such as flexion of the thumb into the palm (top, $41 ;$ bottom, 0$)$. This apraxic syndrome was present both contralateral and ipsilateral to the lesion.

dence was left of the symptoms described above, except occasionally they were seen in SMX-2.

However, both SMX-1 and -2 had long-lasting difficulties with the bimanual coordination task. Preoperatively, both animals would reach out almost simultaneously with both hands, the preferred hand above the plate and leading the movement and the other hand underneath. The index of the preferred hand pushed the bait down while the other hand was cupped underneath (Fig. 6, top). During the reaching movement, either hand would assume a posture anticipating the appropriate movement, the preferred hand showing extension of the index with flexion of the other fingers, the other hand supination and semiflexion of the fingers and thumb.

Although the basic nature of the deficit was the same in both animals, the postoperative course of events was different in each of them. SMX-1 displayed the deficit consistently, without much variation, for over 1 year of testing, whereas SMX-2 developed a number of "trick" movements in an attempt to circumvent the deficit. Therefore, the case histories of these two monkeys will be presented separately. 

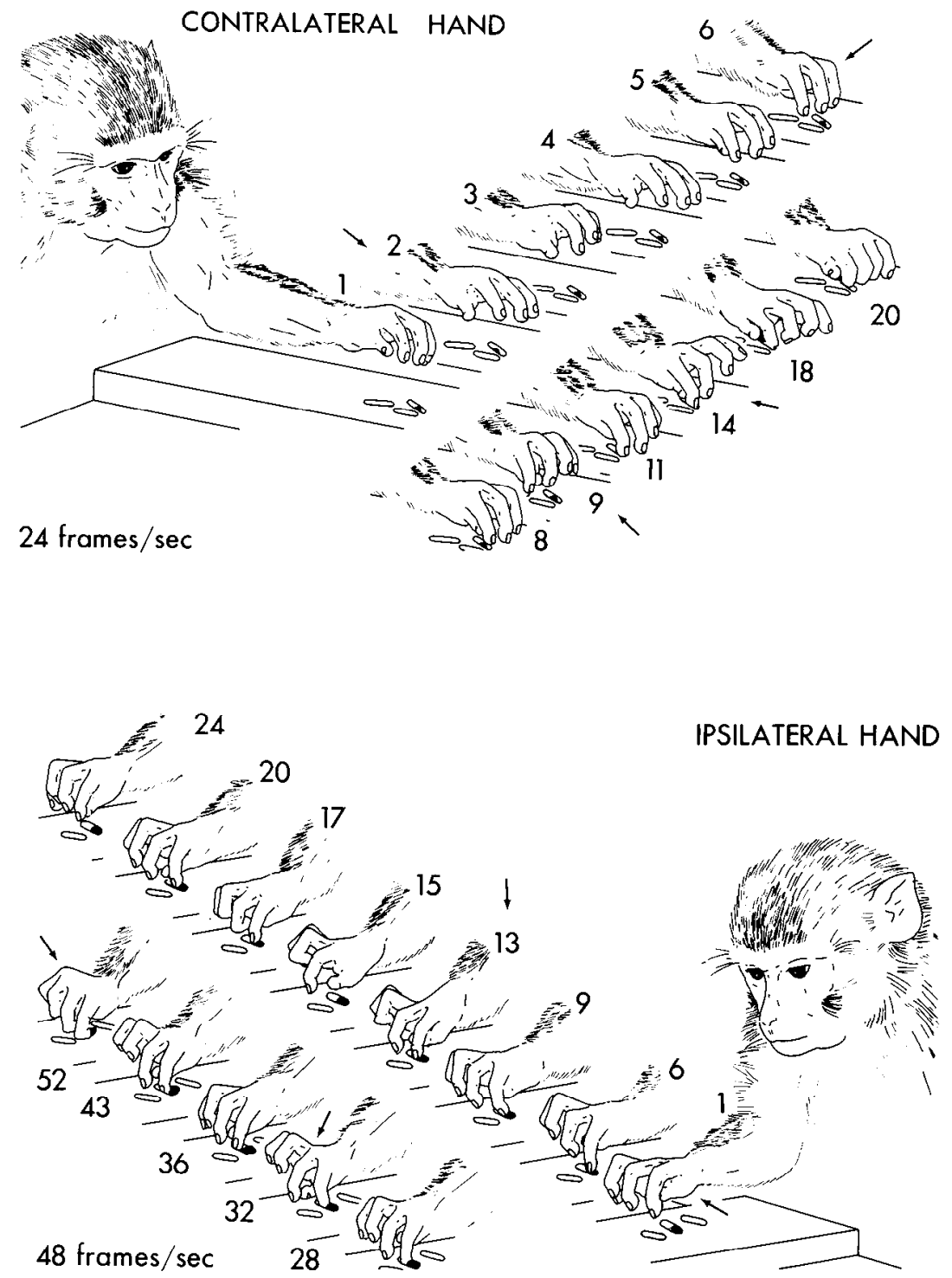

Figure 4. Similar deficits as seen in Figure 3 shown here in monkey SMX-1 with a right SMA ablation, contralateral to the nonpreferred hand, 2 weeks postoperatively. Changes in the movement sequence may occur in as little as $40 \mathrm{msec}($ top, 1 to 6 : flexion and extension with abduction of the fingers, and folding of the thumb into the palm of the hand in 6 ). On casual inspection, the movement performance appeared to be almost normal, but frame-by-frame analysis of movie films and videotapes showed the apraxia. These symptoms were transient and disappeared within 2 to 3 weeks.

Bimanual coordination after an SMA lesion opposite the nonpreferred hand (SMX-1). During the first 2 weeks postoperatively, the deficits seen in the distal and proximal extremity during the unimanual task were also present in the bimanual coordination test. Pushing of the index of the preferred hand into a hole was often clumsy, and associated movements of the other fingers strengthened this impression. The nonpreferred hand frequently was not placed immediately under the hole selected by the leading hand but just next to it, and a correction movement had to be made. Instead of being cupped underneath, anticipating the drop of the bait, the hand showed clumsy finger postures or was pressed flat against the plate. During the second week after surgery, these signs disappeared and were replaced by another type of deficit which persisted throughout the postoperative period of over 1 year. Pushing with the preferred hand became well coordinated and closely resembled the monkey's normal behavior. However, the other hand did not show such recovery: instead, its performance became more and more similar to that of the preferred hand, showing extension of the index and flexion of the other fingers. In this way, the hands would show mirror-type responses on either side of the plate. At times, the nonpreferred hand actually pushed from above and the other from below (Fig. 7, middle). Depending on the outcome of this conflict, either hand could retrieve the bait, and often the nonpreferred hand would move to the next hole first.

From these data it appears that in a bimanual coordination task after a unilateral SMA ablation the hands tend to behave in a similar manner instead of sharing the task between them.

Bimanual coordination after an SMA lesion opposite 
Monkey M-9

HAND TRAJECTORIES

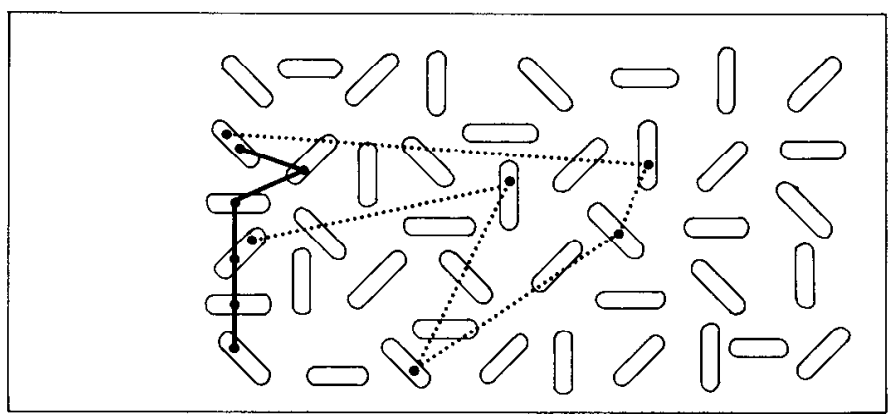

Contralateral hand

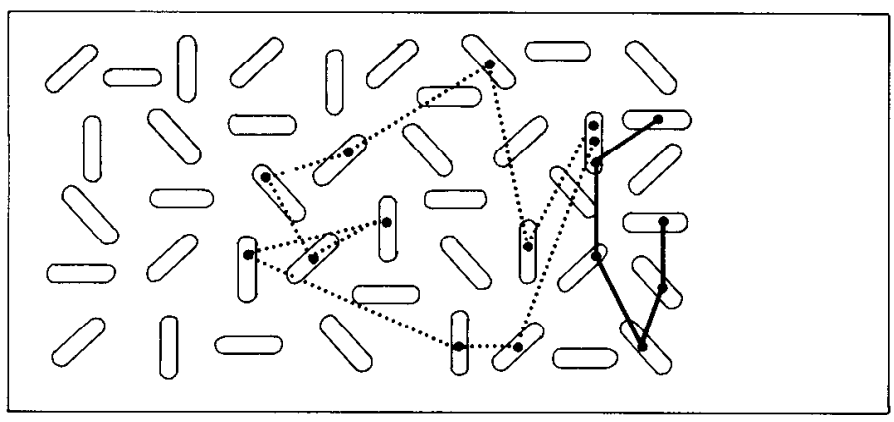

Ipsilateral hand

\section{Preoperatively}

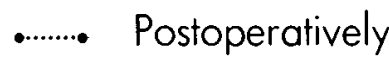

Figure 5. Trajectories of the hands in monkey M-9, preoperatively (solid lines) and 1 week after a right SMA ablation (dotted lines), during a sequence of food retrieval movements. After the lesion, the trajectories became more erratic, which may reflect a disturbance of proximal movement in addition to that seen in the distal movements (Fig. 3). See also Figure 4, top, 6 to 11 , where the monkey did not immediately place its hand on the baited slot.

the preferred hand (SMX-2). In the first postoperative week, this animal did not use the contralateral extremity on the unimanual task, but during the bimanual task it would sometimes reach out with this arm. Nevertheless, usually the nonpreferred hand was now employed in pushing from above, but in a clumsy manner, either because of the deficits in the control of the fingers or because of its relative lack of experience in this movement. In these circumstances the other hand would not be used to catch and the baits were lost. During the second postoperative week, use of the preferred hand became more and more frequent, and a picture similar to that seen in SMX-1 began to emerge, i.e., the two hands making similar movements. In SMX-2, where the "normal" hand was the catching one, these movements took a different form. Both hands reached out, one on either side of the plate, but with little differentiation of posture and with all fingers semiflexed. Rather than pushing simultaneously, as was seen in SMX-1, each hand would make an attempt to lift or pull the bait out of the hole, and successful retrieval was rare. Alternatively, the hand on top of the plate (the preferred hand, contralateral to the lesion) would try to lift the bait out while the other hand was held relatively steady in a catching posture underneath. Gradually, the animal developed a number of strategies to circumvent its difficulties. In one of these, the two hands would reach out above the plate and the index of the nonpreferred hand would be inserted into a hole and push the bait halfway down. This hand was then brought under the plate and the preferred hand completed the push, bringing the food down into the palm of the nonpreferred, catching hand, which might be pulling on the bait as well (Fig. 6). A second strategy, and the one which was adopted most frequently during the postoperative course, was that the animal reached out with one hand on either side of the plate and passively extended the index of the hand on top by resting the tip of this finger on the edge of a hole and pulling the finger a few millimeters backwards, after which a pushing movement followed. Again the hand underneath would often be actively engaged in pulling and dislodging the emerging bait rather than being used in a passive way as a receptacle. With practice, these movements came to be executed so quickly that the behavior resembled the preoperative performance, especially since, once the index had been passively extended, this posture could be maintained over a number of pushes. In contrast to the normal behavior, however, the hand below the plate continued to be actively used in retrieving the food.

In summary, in this animal with a lesion of SMA opposite the preferred hand the nature of the deficit during the bimanual task was initially identical to that seen in SMX-1 with the lesion opposite the nonpreferred hand, namely, similar movements of either hand, but SMX-2 developed a number of "tricks" to mask the deficit.

\section{Effects of a unilateral ablation of the premotor area}

It is possible that the deficits described above were not the result of damage to the SMA but, rather, were a general consequence of a lesion of part of cytoarchitectonic area 6 . Therefore, animals with SMA lesions were compared with an animal (PMX-1) in which a large lesion was made in the lateral part of area 6 , the premotor area, at the level of the arcuate sulcus (Fig. 1). Since the effects of an SMA lesion were most pronounced when the ablation was made opposite the nonpreferred hand, the premotor area lesion was made accordingly.

Like the SMX monkeys, PMX-1 showed little or no change in its general behavior. No paresis occurred, and locomotion, climbing, resting posture, grooming, and feeding were unaffected. On the unimanual task, retrieving food from the slots, mild transient clumsiness was present bilaterally, but it was less pronounced than that seen after the SMA lesions.

On the bimanual task, the animal showed some difficulty in the first few postoperative days, presumably because of the clumsiness seen during the unimanual task. However, in clear contrast to the SMX monkeys, after this period, the test was performed skillfully in a manner indistinguishable from the preoperative performance. For this reason, the deficit seen in the SMA- 


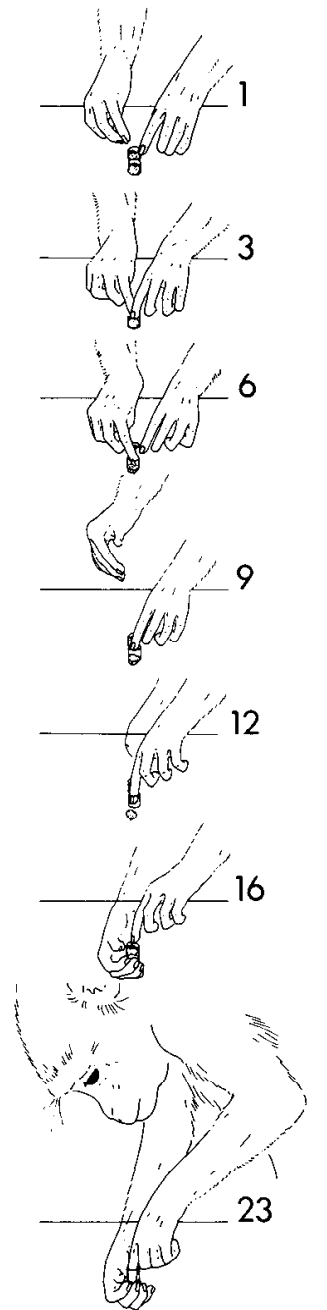

SMX-2 6 months PO

Figure 6. Example of "trick" movements used by monkey SMX-2 with a lesion of the right SMA, opposite the preferred hand, to circumvent the bimanual coordination deficit, seen here 6 months postoperatively. Film speed was 24 frames/sec. Both hands reached out above the plate, and the unaffected right hand initiated the push $(6)$. The left hand then completed the push while the right hand was being brought under the plate to catch the bait. Although on this trial the bait was lost (12), the left hand moved to another hole without change in hand posture (16) and pushed the food down while the right hand was actively engaged in pulling the emerging food from below (23).

ablated monkeys appears to be a genuine consequence of that lesion rather than a general effect of area 6 damage.

\section{Effects of subsequent section of the corpus callosum}

The lasting and basic deficit after a unilateral lesion of SMA appears to be a tendency of the two hands to behave in a similar manner during a bimanual coordination task, instead of sharing the task's components. It may be concluded that the intact SMA now influences not only the motor control systems within the ipsilateral hemisphere, in particular the premotor and primary motor areas, but also those of the contralateral hemisphere in an identical manner. Anatomically, connections of
SMA with the contralateral cortical motor areas have been well established, and these connections travel via the corpus callosum (DeVito and Smith, 1959; Karol and Pandya, 1971; Pandya and Vignolo, 1971). Therefore, section of the corpus callosum might alleviate the bimanual coordination deficit by cutting off this flow of instructions. To test this hypothesis, the corpus callosum was seclioned completely in SMX-1 and -2 and, as a control, in PMX-1 as well.

Effects of callosal section in SMA-lesioned animals. These were seen immediately postoperatively, on the first day, and perhaps were most striking in SMX-1, which until then had continued to show similar movements of either hand (Fig. 7, middle). On the first postoperative day, this behavior had disappeared almost completely. Most of the time, as during normal performance, the preferred hand pushed while the other hand was cupped underneath (cf. Fig. 7, top). The latter hand was occasionally used in pushing from below, but then the other hand would pick up the bait on top of the plate. Simultaneous pushes at the same hole were never seen, although sometimes they occurred at different holes. Over the next few days, these responses, as well as isolated pushes from below with the nonpreferred hand, occurred less and less frequently; 2 weeks after the callosal section, the animal's performance had become like that seen normally, before the SMA lesion, with the two hands sharing the task (Fig. 7, top and bottom).

Monkey SMX-2, which seemed to have compensated for the effects of the SMA lesion by using "tricks" to the extent that its performance upon casual inspection looked like that of the normal monkey, also improved following callosal section. The immediate postoperative effects were similar to those described for SMX-1, and the animal's eventual performance likewise became like that seen before the first operation. Comparison of the films of the bimanual performance before and after callosal section accentuated the nature of the tricks used by this monkey. "Half-pushes" by the nonpreferred hand, passive extension of the index of the preferred hand, and grasping and pulling movements of this hand below the plate had disappeared. As before the SMA lesion, the preferred hand took the more active role while the other hand was essentially passive and was held cupped underneath.

From the above it may be concluded that since callosal section abolishes the bimanual coordination deficit resulting from SMA lesions, this deficit may indeed be the result of the outflow of the intact SMA influencing also the motor systems of the contralateral hemisphere.

Although callosal section relieved the bimanual coordination deficit, the deficits in the control of finger movements seen immediately after the initial SMA lesion during the use of an individual hand returned. Again, symptoms were bilateral and transient. Also, they were not as obvious, even with careful analysis of the records, as when seen after the initial lesion. This suggests that at least part of the compensation for the initial deficit may have taken place through pathways not involving the corpus callosum.

Effects of callosal section in a PM-lesioned animal. The bimanual coordination of PMX-1 was not affected by a 

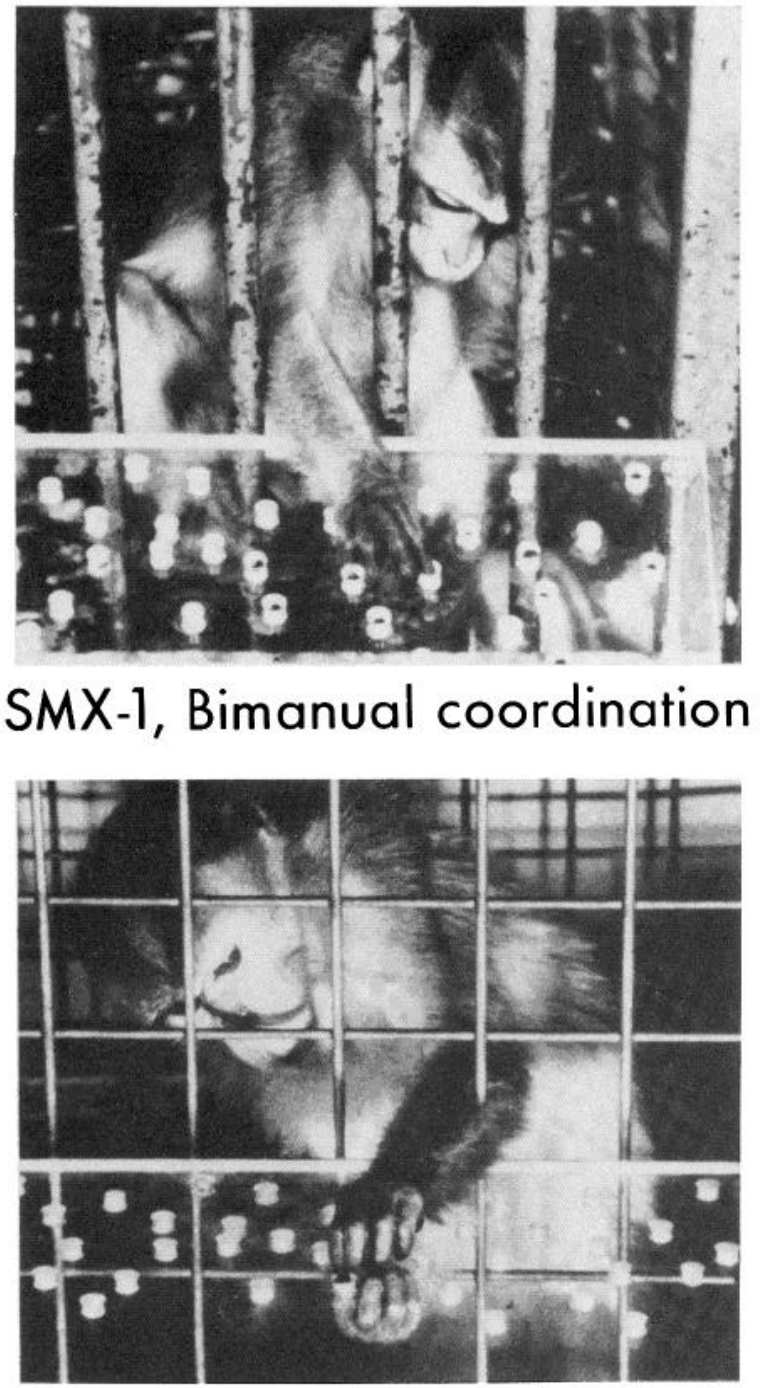

\section{Right SMA lesion, 1 year PO}

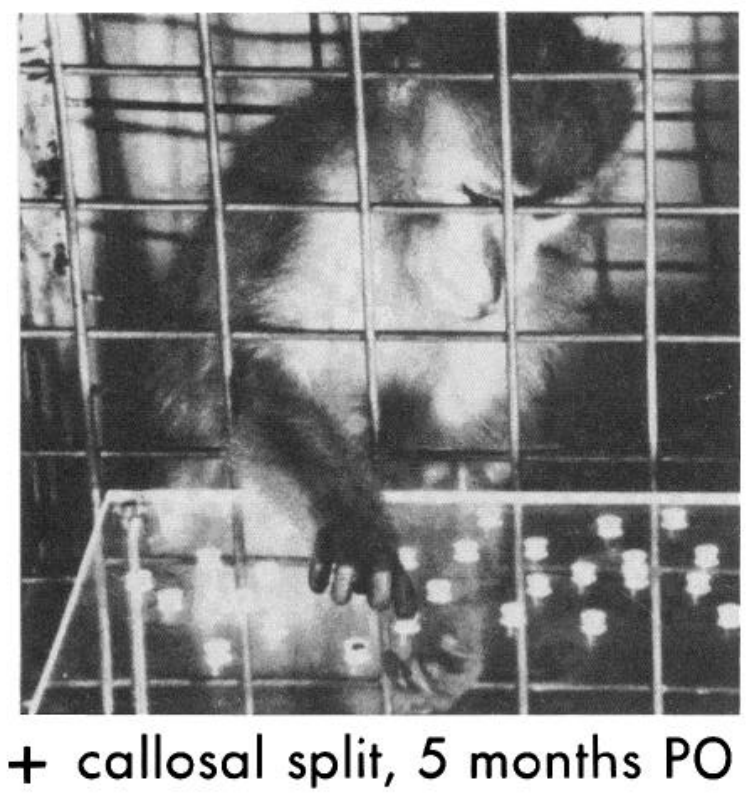

Figure 7. Photographs taken from individual movie frames showing bimanual coordination of SMX-1 before surgery (top), 1 year after a right SMA lesion (middle), and 5 months after lesion of the premotor area, and callosal section did not change this.

\section{Discussion}

In the monkey, a unilateral lesion of SMA did not produce paresis or otherwise affect the animal's general behavior. A bilateral and transient deficit could be observed during tests of control of relatively independent finger movements. However, a lasting deficit was found during the execution of a bimanual coordination task where the two hands tended to behave in a similar or identical manner rather than sharing the work. This deficit was specific to a lesion of SMA since a lesion of the lateral part of area 6 , the premotor area, failed to elicit it. Section of the corpus callosum abolished the deficit on the bimanual task but produced a transient reappearance of the bilateral deficit in relatively independent finger movements.

A dramatic picture of transient, global akinesia and mutism after unilateral SMA excision in man has been described (Laplane et al., 1977; Damasio and Van Hoesen, 1980); but in monkeys studies of SMA ablation in general have reported a lack of symptoms (DeVito and Smith, 1959; Coxe and Landau, 1965), although some have described a transient, contralateral grasp reflex (Penfield and Welch, 1951; Travis, 1955; Smith et al., 1981). Only Travis (1955) reported lasting changes: an increase in muscle tone, especially of the shoulder and elbow flexors.

It is possible that involvement of the anterior cingulate gyrus (area 24) in addition to damage to SMA may produce more severe and longer lasting deficits (Glees et al., 1950; Sutton et al., 1974; C. Brinkman, unpublished observations). In man, the SMA excisions involved at least part of area 24 (Laplane et al., 1977).

The only clear short-term consequence of SMA ablation in the present study was a fragmentation of the normal pattern of relatively independent finger movements required to retrieve a food morsel from a narrow slot. Since the animals did not show any signs of primary motor disturbance in the upper limbs, this defective coordination in purposeful movement may be considered as a form of apraxia (Hecaen and Albert, 1978). No reports exist of apraxia associated with SMA lesions in monkeys, although Gentili et al. (1981) noticed some "clumsiness" of the hands in squirrel monkeys. Using some of Travis's (1955) animals, Hamuy (1956) found a slight impairment on only one of a range of tests of skilled movements, but she ascribed this to the shoulder and elbow muscle hypertonia which interfered with the monkey's movements to turn a crank.

additional callosal section (bottom). In the normal monkey, the two hands shared the task with the index of the preferred hand pushing the bait from the top of the plate while the other hand was cupped underneath. After the SMA lesion, the hands tended to behave in a similar or identical manner, in this case, both pushing the bait from either side, i.e., behaving in a way typical of the preferred hand. Since the lesion was contralateral to the nonpreferred, left hand, it seemed that the intact SMA now controlled both hands, the left hand presumably through pathways involving the corpus callosum. In keeping with this hypothesis, subsequent callosal section restored the normal action of the left hand. 
In man, some transient movement disturbance may be found in association with an infarct of SMA (Masdeu et al., 1978).

The apraxia seen in five monkeys here, as a disturbance of an elaborate reaching and food retrieval movement, points to the importance of SMA in the execution of complex movements. The bilaterality of the deficit is in agreement with the bilateral changes in activity of the area, especially in relation to such movements (Brinkman and Porter, 1979; Roland et al., 1980). This same bilaterality may explain the disappearance of the apraxia since the intact SMA might be able to compensate for the loss of the other. This compensation may be through cortico-cortical connection with the motor areas of the lesioned hemisphere through the corpus callosum (DeVito and Smith, 1959; Karol and Pandya, 1971; Pandya and Vignolo, 1971) or through bilateral subcortical projections, such as to the basal ganglia (Jones et al., 1977), to the red nucleus (Kuypers and Lawrence, 1968; Hartmann-Von Monakow and Akert, 1979), or to the brainstem (Kunzle, 1978). The present data suggest that the transcallosal pathway is the more important one after the initial lesion. The evidence for this is 2 -fold. First, section of the corpus callosum resulted in a return of the apraxia. Second, as has been pointed out above, normal monkeys, when tested on the board with the slots, show a difference between hands in the proficiency with which they retrieve the baits, and this difference was the reason for the distinction of a preferred and a nonpreferred hand. The preferred hand shows a faster and better coordinated opposition movement of the index and thumb and less associated movements of the other fingers. In SMX-1, with a lesion contralateral to the nonpreferred hand, recovery of the apraxia actually led to an improvement of this hand, even compared to its preoperative performance, especially with regard to the associated movements. As a consequence, this hand now resembled the preferred hand. In SMX-2, with a lesion contralateral to the preferred hand, a hint of the reverse was seen: opposition movements appeared less smooth, with thumb movements most affected. Section of the corpus callosum re-established the difference between the hands. These patterns of recovery are consistent with the idea that the intact SMA may influence movements of the hand contralateral to the lesion by way of the corpus callosum.

In human patients with SMA excision, movement disturbances seen immediately postoperatively likewise disappeared, and a deficit persisted only when simultaneous but different movements of the two hands were required (Laplane et al., 1977). This deficit may be the equivalent of the long-lasting bimanual coordination deficit in the monkey. This deficit took the form of similar movements of both hands and was more pronounced in SMX-1 with a lesion opposite the nonpreferred hand. In fact, the animal behaved as if it had two preferred hands, as was also suggested by its performance on the slot board. The deficit in the other monkey was less obvious. In man, SMA excision in the nondominant hemisphere resulted in a more severe deficit during bimanual movements (Laplane et al., 1977). If the bimanual deficit is due to the intact SMA imposing, as it were, an identical motor "program" on both hemispheres, it seems that it is more difficult to "override" this program if it originated in the hemisphere contralateral to the preferred hand, or in the dominant hemisphere in the human cases. In humans with callosal section, for instance, the more powerful influence of the motor systems of the dominant hemisphere can be seen in tasks in which these systems can easily override control of the right hand by the nondominant, right hemisphere (Sperry et al., 1969). Hemispheric specialization in monkeys is still a controversial subject, as is their hand preference (Warren, 1977). However, although simple reaching tasks may not reveal hand preferences in monkeys, tests requiring skilled, relatively independent movements of the fingers do (C. Brinkman, unpublished observations; cf. Engele et al., 1982). If such hand preference is taken into account, it seems that some hemispheric predominance may exist in the monkey in relation to motor control, if only with regard to SMA. Recent evidence suggests that this may also apply to the second somatosensory area $\mathrm{S}$ II (Garcha et al., 1982).

The nature of the bimanual coordination deficit suggested that the intact SMA "programmed" the motor systems of both the ipsilateral and contralateral hemispheres. In accordance with this hypothesis, the bimanual deficit disappeared completely and immediately after section of the corpus callosum. Callosal section in the otherwise intact individual seems to create two more or less independent "action centers," as evidenced by mirror-type responses (Lehman, 1972; Brinkman and Kuypers, 1973), and such responses were seen also in the SMA-lesioned animals.

Two more points are of interest with regard to the callosal section. First, the transient bilateral apraxia was less severe than that seen after the initial SMA lesion and was transient. This suggests that, although the corpus callosum may have played a role in the recovery from the initial apraxia, subcortical mechanisms must also be involved since these would be responsible for recovery after callosal section. Second, although the bimanual coordination returned to a level which is seemingly normal and like that seen in the unoperated monkey, nevertheless, one hand's performance is accomplished without SMA. Other areas within the lesioned hemisphere seem able to compensate for this and achieve normal movement performance. In this respect, the premotor area which has also been cut off from transcallosal "instruc. tion" may be of importance in view of its close connectivity with the primary motor area (Matsamura and Kubota, 1979; Muakassa and Strick, 1979).

The lack of any residual deficit on either the unimanual or a bimanual task used in the present study leaves unanswered the question of the role of SMA in normal movement execution in the intact animal or in man. However, some inferences can be made. In man, during performance of a complex movement, SMA activity, as measured by changes in regional cerebral blood flow, increases more than any other area (except the primary motor area) bilaterally (Roland et al., 1982), and it is the only area to show a high level of activity during thinking about the performance of such movement (mental rehearsal), when again the changes occur bilaterally (Roland et al., 1980). In the monkey performing a learned motor task, neurons in SMA are modulated in their 
activity, regardless of whether the animal uses the contralateral or the ipsilateral limb. Moreover, the discharge patterns of such cells for each hand are very similar (Brinkman and Porter, 1979). The close interconnection of SMA with the motor areas in the contralateral hemisphere (Pandya and Vignolo, 1971) and the fact that, after removal of one SMA, both limbs seem to receive similar motor "programs" suggest that, in the intact animal and in man, discharges from one SMA may act to inform the other hemisphere, in particular SMA, of intended and/or ongoing movements. In the case of unimanual tasks, this may serve to prevent the duplication of responses, whereas in bimanual tasks it may aid in the selection of appropriate movement responses by the other hemisphere or perhaps by the other SMA only. The conveying of such discharges has been suggested as a function of the corpus callosum (Sperry et al., 1969; Preilowski, 1972), and it may be reflected in the bilateral activation of SMA in man and in the bilaterality of neuronal activity in the behaving monkey. When recordings were made from SMA on one side, the pattern of modulation was similar for each hand, but there could be differences in the timing of the increases and decreases in activity. With respect to hand preference, for instance, the modulation was prolonged somewhat in accordance with the usually slower movements of the nonpreferred hand, even if this hand was ipsilateral to the recording site. It is conceivable that this slower pattern may represent discharges from the other SMA reaching the recording site through the callosum (Brinkman and Porter, 1979).

In summary, the present results imply a role for SMA in the programming of complex movements, especially during a bimanual coordination task, a role specific to SMA since lesions of other parts of the "motor association" cortex of area 6 did not affect this behavior. In the normal individual, SMA may be the source of discharges which are aimed at the motor systems of the other hemisphere and which travel through the corpus callosum. More experiments will be needed to specify how SMA programs complex movements. Nevertheless, the present data suggest that one of its functions may be to let one hand know what the other hand is doing.

\section{References}

Banknieder, A. R., J. M. Phillips, K. T. Jackson, and S. I. Vinal, Jr. (1978) Comparison of ketamine with the combination of ketamine and xylazine for effective anesthesia in the rhesus monkey (Macaca mulatta) Lab. Anim. Sci. 28: $742-745$.

Brinkman, C. (1981) Lesions in the supplementary motor area interfere with a monkey's performance of a bimanual coordination task. Neurosci. Lett. 27: 267-270.

Brinkman, C. (1982) Callosal section abolishes bimanual coordination deficit resulting from supplementary motor area lesion in the monkey. Soc. Neurosci. Abstr. 8: 734.

Brinkman, C., and R. Porter (1979) Supplementary motor area in the monkey: Activity of neurons during a learned motor task. J. Neurophysiol. 42: 681-709.

Brinkman, J., and H. G. J. M. Kuypers (1973) Cerebral control of contralateral and ipsilateral arm, hand and finger movements in the split-brain rhesus monkey. Brain 96: 653-674.

Coxe, W. S., and W. M. Landau (1965) Observations upon the effect of supplementary motor cortex ablation in the monkey. Brain 88: 763-772.

Damasio, A. R., and G. W. Van Hoesen (1980) Structure and function of the supplementary motor area. Neurology 30: 359.

DeVito, J. L., and O. A. Smith, Jr. (1959) Projections from the mesial frontal cortex (supplementary motor area) to the cerebral hemispheres and brainstem of the Macaca mulatta. J. Comp. Neurol. 111: 261-277.

Engele, H.-C., B. Preilowski, M. Reger, and E. Weber (1982) Handedness and cerebral asymmetry in non-human primates. Int. J. Primatol. 3: 279.

Garcha, H. S., G. Ettlinger, and J. J. Maccabe (1982) Unilateral removal of the second somatosensory projection cortex in the monkey: Evidence for cerebral predominance? Brain 105: $787-810$.

Gentili, F., R. Tasker, and R. N. Shanlin (1981) Quantitative assessment of muscular hypertonia resulting from cortical lesions in primates. Neurosurgery $9: 48-55$.

Glees, P., J. Cole, C. W. M. Whitty, and H. Cairns (1950) The effects of lesions in cingular gyrus and adjacent areas in monkeys. J. Neurol. Neurosurg. Psychiatry 13: 178-190.

Goldberg, G., N. H. Mayer, and J. U. Toglia (1981) Medial frontal cortex infarction and the alien hand sign. Arch. Neurol. 38: 683-686.

Hamuy, T. P. (1956) Retention and performance of "skilled movements" after cortical ablations in monkeys. Bull. Johns Hopkins Hosp. 98: 417-444.

Hartmann-Von Monakow, K., and K. Akert (1979) Projections of the precentral and premotor cortex to the red nucleus and other midbrain areas in Macaca fascicularis. Exp. Brain Res. 34: 91-105.

Hecaen, H., and Albert, M. L. (1978) Human Neuropsychology, p. 90, John Wiley \& Sons, New York.

Jones, E. G., J. D. Coulter, H. Burton, and R. Porter (1977) Cells of origin and terminal distribution of corticostriatal fibers arising in the sensorimotor cortex of monkeys. J. Comp. Neurol. 173: 53-80.

Karol, E. A., and D. N. Pandya (1971) The distribution of the corpus callosum in the rhesus monkey. Brain 94: 471-486.

Kunzle, H. (1978) An autoradiographic analysis of the efferent connections from premotor and adjacent prefrontal regions (areas 6 and 9) in Macaca fascicularis. Brain Behav. Evol. 15: $185-234$.

Kuypers, H. G. J. M., and D. G. Lawrence (1968) Cortical projections to the red nucleus and the brainstem in the rhesus monkey. Brain Res. 4: 151-188.

Laplane, D., J. Talairach, V. Meininger, J. Bancaud, and J. M. Orgogozo (1977) Clinical consequences of corticectomies involving the supplementary motor area in man. J. Neurol. Sci. 34: f301-314.

Lehman, R. A. W. (1972) Constancy of hand preference following forebrain bisection in monkeys. Neurology 22: 763-769.

Macpherson, J. M., C. Marangoz, 'T. S. Miles, and M. Wiesendanger (1982) Microstimulation of the supplementary motor area (SMA) in the awake monkey. Exp. Brain Res. 45: 410416.

Mark, R. F., and R. W. Sperry (1968) Bimanual coordination in monkeys. Exp. Neurol. 21: 92-104.

Masdeu, J. C., W. C. Schoene, and H. Funkenstein (1978) Aphasia following infarction of the left supplementary motor area. Neurology 28: 1220-1223.

Matsamura, M., and K. Kubota (1979) Cortical projection to the hand-arm motor area from post-arcuate area in macaque monkeys: A histological study of the retrograde transport of horseradish peroxidase. Neurosci. Lett. 11: 241-246.

Muakassa, K. F., and P. L. Strick (1979) Frontal lobe inputs to primate motor cortex: Evidence for four somatotopically 
organized "premotor" areas. Brain Res. 79: 176-182.

Napier, J. R. (1956) The prehensile movements of the human hand. J. Bone Joint Surg. 388: 902-913.

Orgogozo, J. M., and B. Larsen (1979) Activation of the supplementary motor area during voluntary movements in man suggests it works as a supramotor area. Science 206: 847850.

Pandya, D. N., and L. A. Vignolo (1971) Intra- and interhemispheric connections of the precentral, premotor and arcuate areas in the rhesus monkey. Brain Res. 26: 217-233.

Penfield, W., and K. Welch (1951) The supplementary motor area of the cerebral cortex. Arch. Neurol. Psychiatry 66:289317.

Preilowski, B. F. B. (1972) Possible contribution of the anterior forebrain commissures to bilateral motor coordination. Neuropsychologia 10:267-277.

Roland, P. E., B. Larsen, N. A. Larsen, and E. Skinhфj (1980) Supplementary motor area and other cortical areas in organization of voluntary movements in man. J. Neurophysiol. 43: $118-136$.

Roland, P. E., E. Meyer, T. Shibasaki, Y. L. Yamamoto, and C. J. Thompson (1982) Regional cerebral blood flow changes in cortex and basal ganglia during voluntary movements in normal human volunteers. J. Neurophysiol. 48: 467-480.

Smith, A. M., D. Bourbonnais, and G. Blanchette (1981) Interaction between forced grasping and a learned precision grip after ablation of the supplementary motor area. Brain Res. 222: 395-400.
Sperry, R. W., M. S. Gazzaniga, and J. E. Bogen (1969) Interhemispheric relationships: The neocortical commissures; syndromes of hemisphere disconnection. In Handbook of Clinical Neurology, P. J. Vinken and G. W. Bruyn, eds., Vol. 4, pp. 273-290, Elsevier-North Holland Publishing Co., Amsterdam.

Sutton, D., C. Larson, and R. C. Lindeman (1974) Neocortical and limbic lesion effects on primate phonation. Brain Res. 71: 61-75.

Tanji, J., and K. Kurata (1982) Comparison of movementrelated activity in two cortical motor areas of primates. J. Neurophysiol. 48: 633-653.

Travis, A. M. (1955) Neurological deficiencies following supplementary motor area lesions in Macaca mulatta. Brain 78: 174-201.

Warren, J. M. (1977) Handedness and cerebral dominance in monkeys. In Lateralization in the Nervous System, S. Harnad, R. W. Doty, L. Goldstein, J. Jaynes, and G. Krauthamer, eds., pp. 151-172, Academic Press, Inc., New York.

Wise, S. P., and Tanji, J. (1981) Supplementary and precentral motor cortex: Contrast in responsiveness to peripheral input in the hindlimb area of the unanesthetized monkey. J. Comp. Neurol. 195: 433-451.

Woolsey, C. N., P. H. Settlage, D. R. Meyer, W. Spencer, T. P. Hamuy, and A. M. Travis (1952) Patterns of localization in precentral and "supplementary" motor areas and their relation to the concept of a premotor area. Res. Publ. Assoc. Res. Nerv. Ment. Dis. 30: 238-264. 